\title{
Altered Motor Control in Athletes with Low Back Pain: a Literature Review
}

\author{
${ }^{1}$ Rahman Sheikhhoseini" ${ }^{2}$ Kieran O'Sullivan, ${ }^{3}$ Mohammad Hossein Alizadeh, ${ }^{1}$ Meisam Sadeghisani \\ ${ }^{1}$ Faculty of Sport Sciences, Allameh Tabataba'i University, Tehran, Iran. ${ }^{2}$ Sports Spine Centre, Aspetar Orthopaedic and \\ Sports Hospital, Doha, Qatar. ${ }^{3}$ Department of Health \& Sport Medicine, Faculty of Sport Sciences, University of Tehran, \\ Tehran, Iran. ${ }^{4}$ Faculty of Rehabilitation, Shahid Beheshti University of Medical Science, Tehran, Iran.
}

\begin{abstract}
Low back pain (LBP) is also one of the most common medical conditions in athletes. There is little doubt that patients with LBP use from their body differently than pain free individuals. The purpose of this review was to investigate changes in motor control which may be present in athletes with LBP. The search strategy for this review consisted of an electronic database search of full text in MEDLINE database. 28 studies met the eligibility criteria, most of which were cross-sectional in nature. The studies were analyzed separately according to the specific sports involved. The studies demonstrate that athletes with LBP exhibit a range of MCI in the trunk, lumbopelvic region and lower extremities. However, inconsistencies were apparent between the results. Athletes with LBP demonstrate MCI during functional and non-functional tasks, similar to non-athletes. More studies, especially large prospective studies which control for non-mechanical factors which may also differ among athletes with LBP are required to determine the relationship between LBP and MCI in athletes.
\end{abstract}

KEY WORDS: Low Back Pain, Motor Control, Athletes, Biomechanics, Electromyography, Range of Motion.

\section{INTRODUCTION}

Low back pain (LBP) is the most common musculoskeletal problem in the public health with lifespan prevalence up to $80 \%$ in human populations (1). Although in most cases LBP may recover without any medical intervention, the recurrence rate is highly (2). LBP is also one of the most common medical conditions among gymnastics, football, volleyball and tennis players. Its reported that $20 \%$ of sport related injuries involve the spine $(3,4)$.

Treatment options for athletes with LBP including manual therapy, physiotherapy, medication, surgery. However , none of those appear clearly superior (5). This may be due to the fact that many of these strategies do consider possible underlying mechanisms such as the presence of motor control impairments (MCI) in athletes with LBP (6).

Although the study of the mechanisms and complexity of the changes in motor control associated with LBP has been started recently (7), there is little doubt that people with LBP use from their body differently in comparison to the pain free individuals (6-10). These motor control impairment (MCI) changes may present as a spectrum from subtle changes in muscle coordination to complete avoidance of movement, and from hypermobility to hypomobility in the involved segments (7).

*. Corresponding Author:

Rahman Sheikhhoseini

E-mail: rahman.pt82@gmail.com 
Although alterations in motor control may potentially protect damaged tissues from further injuries in the acute phases via redistribution of load $(8,11)$ persistence of these alterations after recovery of LBP could jeopardize tissue health and restoration of normal function (12).

Recently, special attention has been paid to motor control training for the treatment of LBP (9, 11). Therefore, understanding the MCIs associated with LBP can assist in planning of more effective management of athletes who suffering from LBP. Although different studies have been conducted on MCI in athletes with LBP (13-21) it is necessary to investigate the MCIs in various sports in sports-specific functions separately.

The purpose of this review is to review studies relating to MCI in athletes with LBP in various sports.

\section{MATERIALS AND METHODS}

The search strategy was based on an electronic database search of titles and abstracts. An electronic search was done in MEDLINE without time restriction. Search terms used included: "Back Pain", "Motor Control", "Muscle Activation", Kinematics, "Range of Motion", Athlete, Football, Soccer, Golf, Judo, Volleyball, Gymnastics, Running, Tennis, Cycling, Cricket, Dance and Sport. Only articles that had been published in peer-reviewed journals in the English language were included. Initially, the titles and abstracts were reviewed by the first author. Then, the fifth author rereviewed the search strategy to confirm the accuracy of research. In cases of disagreement on study eligibility a consensus method was used. Only studies that compared athletes with LBP with painfree controls were selected. Thereafter articles were classified based on sports. There were no other inclusion criteria.

\section{RESULTS}

In total 461 records were initially identified through the MEDLINE database. Of these, 105 potentially eligible articles were included based on their title and abstract. After reviewing these 105 potential articles, only 28 articles fulfilled the inclusion criteria. Due to the heterogeneity, the selected articles were classified based on sports. The studies consist of 26 cross-sectional studies, one Experimental longitudinal study and one Stepped-wedge intervention study. The key characteristics of each study are illustrated in Table 1, including gender, participants, subgroups, age, definition of LBP, study design, measurement tools, outcome measures and main results.

Cricket. Two studies examined cricketers, measuring kinematics of bowling (22) and an MRI study of the abdominal wall muscles (23), respectively. The kinematics of bowling technique during the delivery stride in elite Australian female fast bowlers were examined by Stuelcken et al. (2010). The results showed that in female fast bowlers with a history of LBP (HLBP), the thorax was positioned in greater side flexion relative to the pelvis during the delivery stride than among those without LBP. LBP bowlers also performed the delivery stride using a significantly greater range of thorax side flexion relative to the pelvis while there was no correlation between large shoulder counter rotation and HLBP (22). It was proposed that the increased lumbar lateral flexion in those with LBP may insert high stress on the zygapophyseal joint cartilage which may lead to capsular ligaments strain and subsequently elicit the pain symptom (24).

Hides et al. (2008) conducted a crosssectional MRI study to determine differences in muscular activation and symmetry in male fast bowlers with $(n=10)$ and without $(n=16)$ a HLBP during abdominal hollowing test. They found that asymmetry in the size of quadratus lumborum (QL) muscle may be evident in cricket athletes. However, the asymmetry was greatest in the fast bowlers with HLBP. The ability of the LBP group to preferentially activate the transversus abdominis (TrA) muscle compared to the other abdominal muscles was reduced significantly and they couldn't perform the maneuver as well as the painfree group. (23). The increased lateral flexion in athletes with LBP observed by Stuelcken et al. (2010) may be related to these muscular asymmetries.

In summary, it seems that the bowlers with a HLBP perform the bowling and delivery techniques with significantly greater side flexion angle on the dominant side, which may explain the thicker QL muscle ipsilaterally. These findings could be used to regulate the athlete's technique of bowling and delivering and to 
recommend specific exercises to target mentioned muscle imbalances.

Cycling. Two studies among cyclists were eligible (10, 25), measuring movement kinematics and EMG of muscles of lower lumbar during cycling. Burnett et al. (2004) found that cyclists with LBP (flexion pattern) rode with greater lower lumbar spine flexion and rotation and less lumbar flexion and rotation in the upper lumbar spine, though these differences did not reach statistical significance. LBP cyclists also showed a greater asymmetry in superficial lumbar multifidus activity during riding (25). Van Hoof et al. (2012) showed that cyclists with LBP (flexion pattern) displayed significantly greater flexion (near the end range) in the lower lumbar spine during 2 hours of riding, and that lumbar flexion angle did not deteriorate over time. This suggests that MCI is associated with increased lower lumbar flexion angle in cyclists with LBP rather than reduced lumbar muscle endurance (10). Since the bending moments increase near the end ranges, greater stress may be place on sensitive spinal structures in cyclists with LBP and provoke their pain $(26,27)$.

It seems that elite cyclists with LBP (flexion pattern) display greater lower lumbar flexion and rotation and greater asymmetry in superficial lumbar multifidus activity during cycling. It should be kept in mind that both studies specifically only included those with flexion pattern LBP, so it is no surprise that the cyclists had more flexion in the lumbar spine.

Dance. Four studies among dancers were eligible, one of them investigated trunk mechanical properties of stiffness and damping (28), two of them measured muscle thickness of abdominal muscles by means of MRI $(11,29)$ and the other examined the lumbopelvic motor control by means of PBU (16). Gildea et al. (2014) demonstrated that professional dancers with a HLBP had significantly lower trunk damping during trunk movement in response to perturbations, but observed no significant differences in trunk stiffness between dancers with and without LBP (28).

In an observational MRI study, Gildea et al. (2014) examined TrA and IO morphology in professional ballet dancers both at rest and during performing the abdominal muscle "draw in" maneuver. The results showed that there was no any significant difference in TrA and IO thickness between dancers with and without pain. TrA demonstrated significantly less slide in dancers with LBP than in dancers without LBP. The amount of reduction in abdominal cross sectional area in people with LBP was similar to painfree athletes during the performing abdominal "draw in" (29). In another paper by the same research group, they found significantly smaller multifidus muscles size at the lower lumbar levels (11). Roussel et al. (2013) demonstrated that 30\% (7/23) of dancers without a HLBP were unable to contract their TrA muscle correctly in comparison to $63 \%$ (10/16) of dancers with a HLBP(16).

Generally, we can conclude that there is not enough evidence to confirm specific muscle asymmetry in dancers with LBP, which may be due to small sample sizes (29). Although poor lumbopelvic motor control and lower trunk damping are seen in the dancers with LBP, but further studies are needed to clarify the issue.

Football. Three MRI studies, all performed by the same research group, measuring the thickness of the abdominal and pelvic muscles were eligible (13-15). Hides et al. (2010) compared the ability of 43 elite male Australian football players with and without LBP from a single club to contract the abdominal wall. The ability of professional football players with current LBP to draw in the abdominal wall was significantly reduced in comparison to asymptomatic players (14). Moreover, in the second study, this deficit was found to be more evident in the lower abdominal region (13). The final study found that cross sectional area (CSA) of the piriformis muscle was similar in football players with and without current LBP but the typical piriformis adaptation to increased physical demands during the season was affected negatively by LBP. In addition, the risk of sustaining lower limb injuries during the season was significantly higher in the players with smaller piriformis CSA (15).

In summary, a decreased ability to perform the abdominal "draw in" maneuver and smaller piriformis CSA were observed among the elite football players with LBP.

Golf. Seven studies were eligible, two of them examined hip ROM and/or the strength of the core muscles $(21,30)$, two of them examined the 
kinematics of swing or drive movements $(20,31)$, and three of them examined EMG of selected muscles (17-19). Vad et al. (2004) and Murray et al. (2009) reported significantly reduced range of active and passive hip internal rotation in lead hip and reduced lumbar extension $\operatorname{ROM}(21,30)$ in amateur golfers with LBP.

Tsai et al. (2010) reported that male golfers with HLBP demonstrated significantly less isokinetic strength in trunk extension and hip adduction in comparison to age and handicap matched controls. Although the trunk rotation ROM toward the non-lead hip was significantly decreased, they found no significant differences between kinematics and kinetics of the trunk during the golf swing (20). Cole et al. (2014) studied the differences in the crunch factor (CF) _or the combination of the trunk lateral flexion and axial rotation during the swing phase between golfers with and without LBP. However, they found that there was no significant differences between $\mathrm{CF}$ in male golfers with and without LBP (31).

Electromyographic studies demonstrated that low-handicap golfers with LBP had less activity in ES and more activity in External Oblique (EO) muscles. In contrast, ES activity in high-handicap golfers with LBP was greater than asymptomatic golfers, while the golfers performed ball driving (17). However Horton et al. (2001) found no differences between abdominal muscles activation in golfers with and without LBP during the golf swing (18). On the other hand Suter and Lindsay (2001) found that reduced endurance of back extensors was associated with the knee extensors inhibition in golfers with LBP compared to painfree golfers (19).

In general, it seems that reduced internal rotation in the lead hip and reduced lumbar spine extension ROM are present in golfers with LBP. There was inconsistency among the result of kinematic and EMG studies in functional sport specific tasks.

Hockey. One study was eligible (32). Fenety and Kumar (1992) reported that elite female field hockey players with LBP showed significantly lesser spinal extension ROM and total spinal ROM than painfree hockey players. The athletes with LBP also had weaker peak and average eccentric spinal extension torques(32). These findings suggest that the athletes with
LBP had less spinal ROM and the attention should be pay to these discrepancies.

Judo. One study that measured hip internal and external rotation ROM in judo athletes with and without LBP was eligible (33). Almeida et al. (2012) found that athletes with LBP exhibited a significant reduction in active hip internal rotation and active total hip rotation of the non-dominant limb in comparison with the painfree athletes. In addition, reduction in passive internal rotation of the dominant and non-dominant limbs, as well as passive total hip rotation of the non-dominant hip was detected in LBP group compared to painfree group. The LBP group also exhibited significant ROM reduction in active and passive hip internal rotation and active and passive hip total rotation of the non-dominant limb compared to dominant $\operatorname{limb}(33)$.

It seems that judo athletes with LBP show lesser hip internal rotation in both hips and asymmetry in hip ROM is detectable among the athletes.

Running. One study measuring pelvis-trunk coordination and coordination variability during walking and running was eligible (34). Seay et al. (2011) found that coordination in frontal plane in runners with LBP was more in-phase _ a continuous relative phase of $0^{\circ}$ represents "inphase" motion between the pelvis and trunk during walking compared to painfree runners and runners with HLBP displayed intermediate coordination pattern. However during running, both LBP and HLBP athletes displayed more in-phase coordination in the transverse plane compared to painfree runners. During running the painfree runners had greater coordination variability in axial rotation rather than people with LBP and improved LBP runners (34). These alterations may lead to further LBP in the athletes (7).

Tennis. Three studies were eligible, the first measuring EMG (35) and two others by a single research group investigated kinematics and kinetics of the tennis serve movement $(36,37)$. Renkawitz et al. (2006) observed significant neuromuscular imbalance of lumbar ES (based on Integrated EMG measures), as well as lower lumbar ROM in all planes of motion, among those with LBP compared to painfree players. They observed no significant differences in maximal isometric trunk extension strength (35).

Campbell et al. (2014) compared trunk and lower limb kinematics during tennis serves 
between adolescent male tennis players with and without HLBP. The findings suggested that during the drive phase of the tennis serves, the HLBP players exhibited lesser rotation in right lower lumbar spine, rotation of shoulder relative to pelvis and left pelvic tilt and also earlier peak velocity of the right knee. As well as during the forward-swing phase they exhibited greater left rotation of the lower lumbar and pelvis, left side bending of the upper lumbar spine and anterior pelvic tilt (36). In their other study, Campbell et al. (2013) showed that players with HLBP utilized greater left lateral force on the lumbar spine during the drive phase of the tennis serves (37). These findings may be important mechanisms to elicit the pain in the tennis players.

In summary tennis players with a HLBP show significant changes in control of movements that include the followings: muscular imbalance in ES muscles, increased spinal stiffness, and some changes in kinematics of trunk and also, lower limb and lumbar loading during the serves.

Rotation related sports. Four studies were found in which subjects were not selected from specific sports but from various sports that placed repetitive rotational demands on the hip and lumbopelvic region to perform most aspects of the activity (like; tennis, racquet ball, golf, etc,.) (38-41). Scholtes et al. (2009) and Van Dillen et al. (2008) demonstrated that in comparison with painfree people who did not play rotation-related sports, the LBP athletes who played rotation related sports showed significantly greater and earlier rotation of the lumbopelvic region while performing active and passive knee flexion and hip external rotation in the prone position (39, 40). However in another similar study using similar participants Chimenti et al. (2013) found no significant differences between both groups in movement pattern variables (38).

Gombatto et al. (2007) compared painfree athletes with two subgroups (rotation and extension rotation subgroups) of athletes with LBP during trunk side bending in standing. They found that the rotation with extension subgroup exhibited significantly more end-range lumbar motion to the left side than the right, reflecting a significant decrease in the percentage contribution of the lumbar spine motion to total trunk side bending to the left. The rotation subgroup displayed significantly more end-range lumbar spine motion to the right side than to the left side (41).

In summary, there was inconsistency between the results of the studies. One explanation for these findings may be the diversity of participants and sports that subjects were selected from. It seems that classifying the LBP problem in these athletes may be helpful to clarify the issue.

\section{DISCUSSION}

The present review has focused on alterations in motor control among athletes with LBP. The existing studies were classified based on the sports. The existing studies demonstrated that athletes with LBP exhibit a variety of MCI, including alterations in kinematics, kinetics, muscle activation and strength of both the trunk and lower limbs. Several inconsistencies were also reported, possibly due to factors such as: a) athletes with LBP not being classified in more homogenous subgroups (most existing studies except for cycling), b) some studies compared the athletes with non-athletes $(39,40)$, c) the target population in some studies was not selected from specific sport (38-40), d) studies in the same sport examined tasks that were not always sport-specific $(13,15,20,21,30,32,33$, 38-40), e) duration of pain varied between studies, f) some studies did not describe sex differences, g) the role of pain and its various intensity and durations on the MCI remained unclear, and $\mathrm{h}$ ) there are significant differences in assessment methods of different studies.

The current review collected evidence that may have implications for technical remediation in athletes with LBP. The coaches should focus on motions that approach to end range during the sports and try to resolve ROM discrepancies in the athletes with LBP. Based on the presented evidence, the following conclusions can be generated regarding altered motor control in athletes with LBP:

- Cricket athletes with LBP perform delivery stride with greater trunk side flexion between front foot contact and ball release, and also muscle imbalance is present in the lumbopelvic muscles.

- Cyclist with flexion pattern LBP drive with more flexion of the lower lumbar than the controls. Coaches should consider helping such 
athletes to reduce the excessive lower lumbar flexion during riding, through biofeedback (42) or by adjusting the saddle angle (43).

- Dancers with LBP have poorer lumbopelvic motor control strategies.

- The ability of football players with LBP to draw in the abdominal wall decrease in the lower lumbar region.

- Golfers with LBP show reduced range of active and passive internal rotation in the lead hip, reduced the lumbar extension, reduced the lumbar rotation toward the lead hip and altered muscular activation pattern. It seems that focus on hip internal rotation discrepancy in golfers with LBP may be helpful to managing LBP in the athletes $(44,45)$.

- Hockey players with LBP have lesser spinal extension and total spinal ROMs.

- Reduced active and passive hip internal rotation and active and passive hip total rotation of the non-dominant limb is present in Judo athletes with LBP.

- Runners with LBP walk with more in-phase coordination in frontal plan and run with more in-phase coordination in transverse plan.

- Tennis players with LBP display kinematics alterations and increased left lateral force on the lumbar loading during the serves. Neuromuscular imbalance of lumbar ES and reduced lower lumbar ROM is also present in the LBPs. The serving technique of the LBP players should be remediated to avoid excessive movements during forward swing phase and greater left lateral force on the lumbar spine during the drive phase.

- In Rotation related sports there is inconsistency between the results of the studies. It seems that classifying the LBP problem in these athletes may be helpful to clarify the issue.

The cross-sectional nature of most studies means we cannot determine causation, and it is possible these changes are all secondary to pain. The variation between studies makes it hard to compare them. However, we can suggest that athletes with LBP do not seem to adopt postures that are automatically the best or most optimum, and that they may be essentially maladaptive postures and movements. This explains the flexion pattern cyclists staying in flexion during cycling, the cricketers leaning over onto the painful side even more towards end-range as well as the rotation group (reporting pain on rotation) doing more rotation than the painfree group. In other words, athletes do not seem to automatically be assuming the best movement pattern and this might be explained by poor body awareness (46) and poor proprioception (47). We need to acknowledge that psychological factors could explain some MCI too (48), and more studies are needed to clarify the issue in athletes with LBP.

In future studies, LBP problem should be classified in more homogenous subgroups. More studies are required to examine the sport specific techniques in the various sports. The most pain provocative movements during the sports must be selected. Conducting prospective studies to determine the role of the MCI in athletes with LBP in development of further pain and injuries are needed. Further interventional studies also are necessary to clarify the role of the remediated techniques on recurrence and development of future studies.

\section{CONCLUSION}

Athletes with LBP show MCI during performing functional and non-functional tasks, like non-athletes. Comparing the results of current studies together is difficult due to considerable variations in the populations, the measurements taken and the tasks examined. More studies are required to determine the association between LBP and MCI in sport specific tasks.

\section{APPLICABLE REMARKS}

- Athletes with LBP do not seem to adopt postures that are automatically the best or most optimum. This explains the flexion pattern cyclists staying in flexion during cycling, the cricketers leaning over onto the painful side even more towards endrange etc., whereas these faulty postures may exacerbate LBP or result in further pain/injuries, so the athletic trainer should be aware of these poor techniques and plan to resolve the $\mathrm{MCI}$ in athletes with $\mathrm{LBP}$. 


\section{REFERENCES}

1. Walker BF. The prevalence of low back pain: a systematic review of the literature from 1966 to 1998. J Spinal Disord. 2000;13(3):205-17.

2. Balague F, Mannion AF, Pellise F, Cedraschi C. Non-specific low back pain. Lancet. 2012;379(9814):482-91.

3. Andrews JR, Harrelson GL, Wilk KE. Physical rehabilitation of the injured athlete. 4th ed. Philadelphia: Elsevier/Saunders; 2012. p. p.

4. Caine DJ, Maffulli N. Epidemiology of pediatric sports injuries. Basel ; New York: Karger; 2005. v. <1- > p.

5. Petering RC, Webb C. Treatment options for low back pain in athletes. Sports Health. 2011;3(6):550-5.

6. O'Sullivan P. Diagnosis and classification of chronic low back pain disorders: maladaptive movement and motor control impairments as underlying mechanism. Man Ther. 2005;10(4):242-55.

7. Hodges PW, Smeets RJ. Interaction Between Pain, Movement and Physical Activity: Short-term Benefits, Longterm Consequences, and Targets for Treatment. Clin J Pain. 2014.

8. Hodges PW, Tucker K. Moving differently in pain: a new theory to explain the adaptation to pain. Pain. 2011;152(3 Suppl):S90-8.

9. Karayannis NV, Jull GA, Hodges PW. Physiotherapy movement based classification approaches to low back pain: comparison of subgroups through review and developer/expert survey. BMC Musculoskelet Disord. 2012;13:24.

10. Van Hoof W, Volkaerts K, O'Sullivan K, Verschueren S, Dankaerts W. Comparing lower lumbar kinematics in cyclists with low back pain (flexion pattern) versus asymptomatic controls--field study using a wireless posture monitoring system. Man Ther. 2012;17(4):312-7.

11. Gildea JE, Hides JA, Hodges PW. Size and symmetry of trunk muscles in ballet dancers with and without low back pain. J Orthop Sports Phys Ther. 2013;43(8):525-33.

12. Marras WS, Davis KG, Ferguson SA, Lucas BR, Gupta P. Spine loading characteristics of patients with low back pain compared with asymptomatic individuals. Spine (Phila Pa 1976). 2001;26(23):2566-74.

13. Hides J, Hughes B, Stanton W. Magnetic resonance imaging assessment of regional abdominal muscle function in elite AFL players with and without low back pain. Man Ther. 2011;16(3):279-84.

14. Hides JA, Boughen CL, Stanton WR, Strudwick MW, Wilson SJ. A magnetic resonance imaging investigation of the transversus abdominis muscle during drawing-in of the abdominal wall in elite Australian Football League players with and without low back pain. J Orthop Sports Phys Ther. 2010;40(1):4-10.

15. Leung FT, Mendis MD, Stanton WR, Hides JA. The relationship between the piriformis muscle, low back pain, lower limb injuries and motor control training among elite football players. Journal of science and medicine in sport. 2014.

16. Roussel N, De Kooning M, Schutt A, Mottram S, Truijen S, Nijs J, et al. Motor control and low back pain in dancers. Int J Sports Med. 2013;34(2):138-43.

17. Cole MH, Grimshaw PN. Electromyography of the trunk and abdominal muscles in golfers with and without low back pain. Journal of science and medicine in sport. 2008;11(2):174-81.

18. Horton JF, Lindsay DM, Macintosh BR. Abdominal muscle activation of elite male golfers with chronic low back pain. Medicine and science in sports and exercise. 2001;33(10):1647-54.

19. Suter E, Lindsay D. Back muscle fatigability is associated with knee extensor inhibition in subjects with low back pain. Spine (Phila Pa 1976). 2001;26(16):E361-6.

20. Tsai YS, Sell TC, Smoliga JM, Myers JB, Learman KE, Lephart SM. A comparison of physical characteristics and swing mechanics between golfers with and without a history of low back pain. J Orthop Sports Phys Ther. 2010;40(7):430-8.

21. Vad VB, Bhat AL, Basrai D, Gebeh A, Aspergren DD, Andrews JR. Low back pain in professional golfers: the role of associated hip and low back range-of-motion deficits. Am J Sports Med. 2004;32(2):494-7.

22. Stuelcken MC, Ferdinands RE, Sinclair PJ. Three-dimensional trunk kinematics and low back pain in elite female fast bowlers. J Appl Biomech. 2010;26(1):52-61.

23. Hides J, Stanton W, Freke M, Wilson S, McMahon S, Richardson C. MRI study of the size, symmetry and function of the trunk muscles among elite cricketers with and without low back pain. Br J Sports Med. 2008;42(10):809-13.

24. Adams MA. The biomechanics of back pain. 3rd ed. Edinburgh: Elsevier; 2012. p. p.

25. Burnett AF, Cornelius MW, Dankaerts W, O'Sullivan P B. Spinal kinematics and trunk muscle activity in cyclists: a comparison between healthy controls and non-specific chronic low back pain subjects-a pilot investigation. Man Ther. 2004;9(4):211-9. 
26. Adams MA, McNally DS, Chinn H, Dolan P. The clinical biomechanics award paper 1993 Posture and the compressive strength of the lumbar spine. Clin Biomech (Bristol, Avon). 1994;9(1):5-14.

27. Dunk NM, Kedgley AE, Jenkyn TR, Callaghan JP. Evidence of a pelvis-driven flexion pattern: are the joints of the lower lumbar spine fully flexed in seated postures? Clin Biomech (Bristol, Avon). 2009;24(2):164-8.

28. Gildea JE, van den Hoorn W, Hides JA, Hodges PW. Trunk Dynamics Are Impaired in Ballet Dancers with Back Pain but Improve with Imagery. Medicine and science in sports and exercise. 2014.

29. Gildea JE, Hides JA, Hodges PW. Morphology of the abdominal muscles in ballet dancers with and without low back pain: A magnetic resonance imaging study. Journal of science and medicine in sport. 2014;17(5):452-6.

30. Murray E, Birley E, Twycross-Lewis R, Morrissey D. The relationship between hip rotation range of movement and low back pain prevalence in amateur golfers: an observational study. Phys Ther Sport. 2009;10(4):131-5.

31. Cole MH, Grimshaw PN. The crunch factor's role in golf-related low back pain. Spine J. 2014;14(5):799-807.

32. Fenety A, Kumar S. Isokinetic trunk strength and lumbosacral range of motion in elite female field hockey players reporting low back pain. J Orthop Sports Phys Ther. 1992;16(3):129-35.

33. Almeida GP, de Souza VL, Sano SS, Saccol MF, Cohen M. Comparison of hip rotation range of motion in judo athletes with and without history of low back pain. Man Ther. 2012;17(3):231-5.

34. Seay JF, Van Emmerik RE, Hamill J. Low back pain status affects pelvis-trunk coordination and variability during walking and running. Clin Biomech (Bristol, Avon). 2011;26(6):572-8.

35. Renkawitz T, Boluki D, Grifka J. The association of low back pain, neuromuscular imbalance, and trunk extension strength in athletes. Spine J. 2006;6(6):673-83.

36. Campbell A, O'Sullivan P, Straker L, Elliott B, Reid M. Back pain in tennis players: a link with lumbar serve kinematics and range of motion. Medicine and science in sports and exercise. 2014;46(2):351-7.

37. Campbell A, Straker L, O'Sullivan P, Elliott B, Reid M. Lumbar loading in the elite adolescent tennis serve: link to low back pain. Medicine and science in sports and exercise. 2013;45(8):1562-8.

38. Chimenti RL, Scholtes SA, Van Dillen LR. Activity characteristics and movement patterns in people with and people without low back pain who participate in rotation-related sports. J Sport Rehabil. 2013;22(3):161-9.

39. Scholtes SA, Gombatto SP, Van Dillen LR. Differences in lumbopelvic motion between people with and people without low back pain during two lower limb movement tests. Clin Biomech (Bristol, Avon). 2009;24(1):7-12.

40. Van Dillen LR, Bloom NJ, Gombatto SP, Susco TM. Hip rotation range of motion in people with and without low back pain who participate in rotation-related sports. Phys Ther Sport. 2008;9(2):72-81.

41. Gombatto SP, Collins DR, Sahrmann SA, Engsberg JR, Van Dillen LR. Patterns of lumbar region movement during trunk lateral bending in 2 subgroups of people with low back pain. Phys Ther. 2007;87(4):441-54.

42. W. Van Hoof, K. Volkaerts, K. O'Sullivan, Verschueren S, Dankaerts W. Cognitive functional therapy intervention including biofeedback for LBP during cycling. A single case study. Sport en Geneeskunde: the Flemish/Dutch journal of sports medicine 2011;1(4):20-6.

43. Salai M, Brosh T, Blankstein A, Oran A, Chechik A. Effect of changing the saddle angle on the incidence of low back pain in recreational bicyclists. Br J Sports Med. 1999;33(6):398-400.

44. Lejkowski PM, Poulsen E. Elimination of intermittent chronic low back pain in a recreational golfer following improvement of hip range of motion impairments. Journal of bodywork and movement therapies. 2013;17(4):448-52.

45. Reinhardt G. The Role of Decreased Hip IR as a Cause of Low Back Pain in a Golfer: a Case Report. HSS J. 2013;9(3):278-83.

46. Mehling WE, Gopisetty V, Daubenmier J, Price CJ, Hecht FM, Stewart A. Body awareness: construct and selfreport measures. PLoS One. 2009;4(5):e5614.

47. Newcomer KL, Laskowski ER, Yu B, Johnson JC, An KN. Differences in repositioning error among patients with low back pain compared with control subjects. Spine (Phila Pa 1976). 2000;25(19):2488-93.

48. Michael E. Geisser, Michael E. Robinson, Riley JL. Pain beliefs, coping, and adjustment to chronic pain: Let's focus more on the negative. Pain Forum. 1999;8(4):161-8. 